\title{
An Electrical Perspective of the Baroreflex Feedback Mechanism for Heart Rate Control
}

\author{
Lubna Moin $^{1}$, Bhawani Shankar Chowdhry ${ }^{2}$ and Vali Uddin ${ }^{3}$ \\ ${ }^{1}$ National University of Sciences and Technology, NUST-H-12 Islamabad, \\ Pakistan \\ ${ }^{2}$ Mehran University of Engineering and Technology, Jamshooro, Pakistan \\ ${ }^{3}$ Hamdard University, Madinatul Hikmat, Karachi, Pakistan \\ *lubna@pnec.nust.edu.pk; bsc_itman@yahoo.com; vali.udin@hamdard.edu.pk
}

\begin{abstract}
In this research paper an electrical frame work model of baroreflex feedback mechanism is developed. The model proposed can observe and predict the changes in the baroreceptor firing rate as a function of varying blood pressure. It will also follow the changes in the sympathetic and parasympathetic output with varying firing rate of baroreceptor. The new and note worthy in our research paper is the representation of the overall baroreflex mechanism by an electrical circuit having active and passive components which is never discussed before. The baroreceptor is modeled using a combination of linear derivative and a sigmoid function where as the CNS is modeled using combination of first order derivatives and PID controller. The results obtained from the proposed model are validated from the existing physiological data and Joint National Committee (JNC) 8 guidelines for the management of hypertension in adults.
\end{abstract}

Keywords: Baroreceptor mechanism, electrical equivalent, cardiovascular system, central nervous system, modeling and simulation

\section{Introduction}

Baroreflex mechanism is a negative feedback system of the human body to keep the stable arterial blood pressure during changing physiological conditions. It can be thought of as a closed loop system with multiple blocks representing the major physiological variables [2]. This is a short term systemic arterial pressure control carried out by sophisticated multi input multi feedback system [1]. Bararoreceptor mechanism is a short term pressure control and has no long term regulatory mechanism; an instantaneous drop in arterial pressure is sensed by baroreceptor followed by a sequence of events, cause increase in heart rate and cardiac contractility. This drop in pressure also simulates the contraction of the vessels and tends to alter the arterial pressure towards its set value. In this way it provides the rapid negative feedback control of arterial blood pressure. The baroreceptor mechanism has no long term regulatory function. An instantaneous step increase in the carotid sinus pressure is followed by enhanced firing activity in the baroreceptor nerve themselves.

Modeling is one of the basic methods of science used to describe and understand system [2]. The non existence of a complete mathematical model that can provide a valid explanation of the baroreflex mechanism is a major gap [3]. No doubt a great work is done on baroreceptor modeling as discussed in section 2-2; our contribution to the already existing work is the proposal of a complete baroreflex mechanism electrical equivalent. The proposed baroreceptor model is represented by 3 subs models. One defining the afferent path consisting of first order derivative and a sigmoid function [ ], the second sub model is of PID controller with derivative and integral gains. This sub model portrays the CNS Sympathetic and Parasympathetic pathways. The Sympathetic and Parasympathetic 
pathways then further proceed constituting the efferent part. The results obtained showed a good match from the existing literature. The paper is structured as follows. Firstly, the brief description of baroreceptor mechanism is discussed in Section 2.1. The existing models are also briefly discussed in Section 2.2. The proposed model is explained in Section 3. The results and conclusions are discussed in Sections 4 and 5 respectively.

\section{Material and Methods}

\subsection{Baroreceptor Mechanism}

By far the best known of the nervous mechanisms for arterial pressure control is the baroreceptor reflex [20]. Basically this reflex is initiated by stretch receptors called either baroreceptor or pressure receptors. "Feedback Signals" are then sent back through the autonomic nervous system to the circulation to reduce arterial pressure downward toward the normal level (system) [25].

Baroreceptors are spray type nerve endings that lie in the walls of the arteries and are most abundant in the wall of carotid artery and aortic arch. It is called a pressure buffer system and the nerves from the baroreceptors are called buffer nerves [18]. A rise in arterial pressure stretches the baroreceptors and causes them to transmit signals into the CNS. "Feedback" signals are then sent back through the automatic nervous system to the circulation to reduce arterial pressure downwards towards the normal level [15]. Physiologically the baroreceptor system for controlling the atrial pressure can be explained in the following Figure 1.

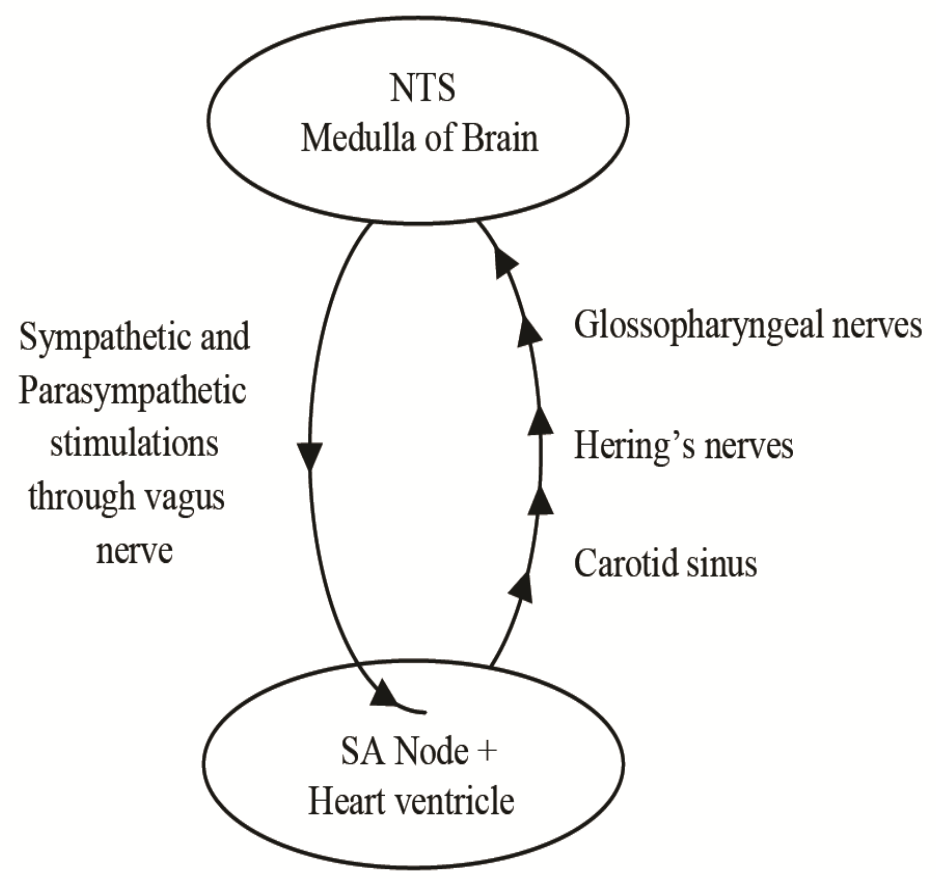

Figure 2.1. Atrial Pressure Control

The above figure illustrates that signals from the carotid baroreceptor are transmitted through small Hering's nerves to the glossopharyngeal nerves in the high neck and then to the NTS Nucleus of Tractus Solitarius in medulla [26]. The Signals in the form of sympathetic and parasympathetic nerves is then transmitted from the brain to heart. The complete baroreceptor mechanism consists of three components [22]. The complete baroreceptor mechanism consists of three Components as shown in Figure 2. 


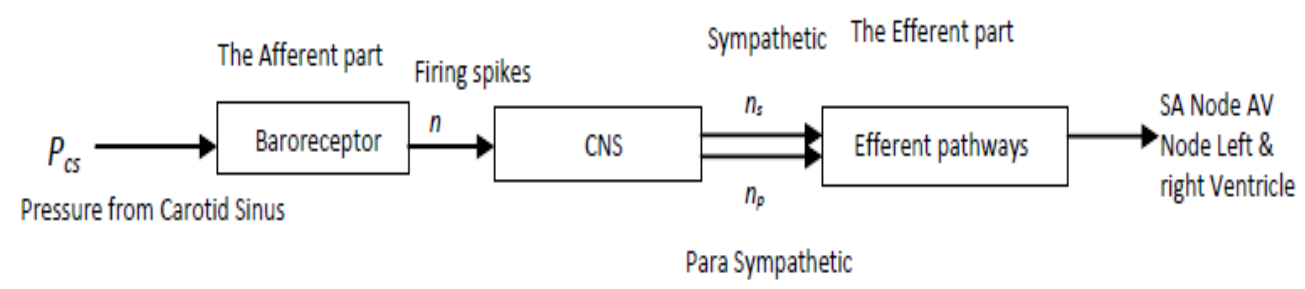

Figure 2.2. Block Diagram for Baroreflex Mechanism

The first component is the afferent part which contains the receptors. The firing rates $\mathrm{n}$ of the receptors are generated by alteration in the arterial pressure $P_{a}$. There are also some non linear behaviors associated with afferent pathway, such as saturation, threshold, and adaptation [9]. The second component is the CNS which generates two nervous activities, the sympathetic $n_{s}$ and the parasympathetic $n_{p}$. The third component called the efferent part consists of pathways to the individual organs in the cardiovascular systems. From the CNS the two nervous activities are transmitted by the efferent pathways to alter heart rate cardiac contractility and vessel constriction [2]. The state of vessel constriction is denoted by $n_{i}$ where i designates the particular organ.

\subsection{Existing Baroreflex Mechanism Mathematical Models}

Many of the great researchers has contributed towards the baroreflex mathematical modeling. Contributions of some of them are very briefly discussed in this section. The regulation of blood pressure by baroreflex was examined in anesthetized dogs by a variety of pressure waveforms were applied to the Carotid Sinus so that the non linear behavior could be thoroughly explored. (William et. al., 1966). Ramachandra et. al., 1972 has proposed a mathematical model describing the relationship between sinus pressure and nerve discharge frequency of the carotid sinus baroreceptor. His research was partly based on the single fibre data obtained by Clarke from the Sinus nerve of day. Like Luciano Bernardi (Luciano et. al., 1997) determined that whether skin blood flow is local or takes part in general regulatory mechanisms. The shape of the auto regulation curve for cerebral blood flow vs pressure was derived by Erzhen Gao (Erzhen Goo 1998). Mauro Ursino (Ursino 1999) in a IEEE transaction described a very famous mathematical model of short term atrial pressure control by the carotid baroreceptor in vagotomized subjects. No doubt it fairly explains the elastance variable of left and right heart, the systemic and pulmonary circulation, the afferent carotid pathway, a central elaboration unit and action of five effecter mechanisms. In the year 2000 J.T Ottesen (J.T Ottesen 2001) provide a comprehensive model of the baroreflex feedback mechanism regulating the heart rate, the contractibility of the ventricle and peripheral vascular resistance, He also modeled the dynamics involved in afferent and efferent pathways. The model proposed by Elisa Magosso and Mauro Ursino (Magosso, Mauro 2001) provides the mathematical model of $\mathrm{CO}_{2}$ effect on cardiovascular system. The new aspects covered in the chemistry behind the process such as the $\mathrm{O}_{2}-\mathrm{CO}_{2}$ interactions at the peripheral chemoreceptor. In 2006 Olufsen, Mette S (Olugen 2006) model the baroreflex regulation of heart rate during orthostatic stress. Their model also reveals that the transfer between the nerve firing blood pressure is non linear and follows a hysteris curve. Baroreflex sensitivity, blood pressure buffering and reference were studied by Hedde Van de Vooren (Hedde Van de Vooren et. al., 2006). The focus on an time variant and non linear characteristics of the baroreflex function and the influence on physiological mechanism on different frequency refilling of blood pressure and heart rate spectrum in studied by M Di Rienzo and his to a 2009 (M Di Rienzo et. al., 2009). In 2011 Alessandro Sil vani (Alessandro Sil vani et. al., 2011) studied cross-circulation function CCF between spate nous fluctuation of heart period and blood pressure as a function of time shift between there variables. A lumped parameter 
model of cerebral blood flow combining cerebral auto regulation and neuro vascular complex was developed by Bart Spronck et. al., in 2012. Adam Mahdi et. al., in 2013 developed a model for predicting baroreceptor firing rate is a function of blood pressure. Their focus was to formulate a family of BR models which could potentially be included in a more comprehensive model of CV systems. The model proposed by Anastasiya (Anastasiya 2013) represented baroreflex model with combination of filter, time delays and gains. His woek was based on (Santos et. al., 2008) approach. In 2014 Vovkodav (Vovkodav and Roman Pasichnyk 2014) presented a modeled of the heart rate and pressure under the influence of physical activity. No doubt the contribution to the field of modeling of baroreflex is marvelous by all the researches but none of them represented the overall mechanism with a electrical equivalent.

\section{Proposed Baroreflex Mechanism Model}

Modeling is one of the basic methods of science used to describe and understand complete system. A physiological model is an abstract representation that enables us to answer questions about the underlying physiological system. The model simulates those aspects of the physiological system that are relevant to the problem under consideration. A modeling method is a graphical, mathematical and actual sets of rule representing the model. No doubt a great work is done on baroreceptor modeling as discussed in Section 2-2; our contribution to the already existing work is the proposal of a complete baroreflex mechanism electrical equivalent. We will firstly illustrate the physiological diagram of baroreflex mechanism in Figure 3.1. Next in Figure 3.2 the flow of heart signal through a block diagram representing, afferent, CNS, parasympathetic, sympathetic and efferent will be developed. Finally, the blocks will be expanded into the detailed electrical components in Figure 3.3 and finally the simulation of the model using multisim and MATLAB.

\section{Baroreceptors}

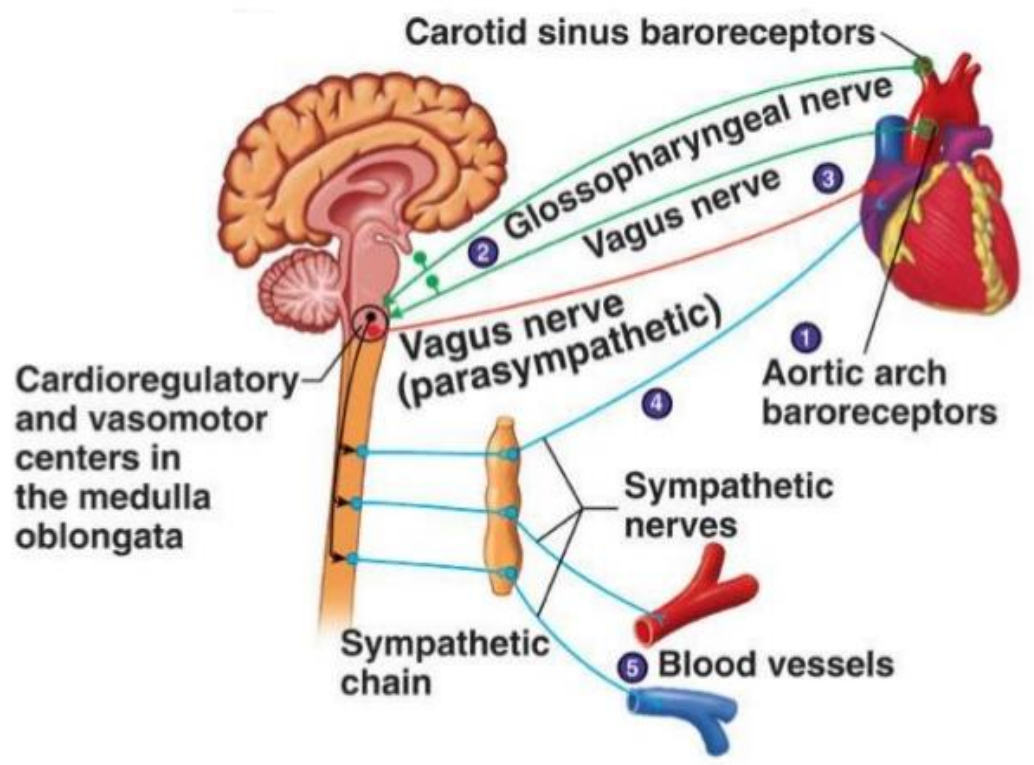

Figure 3.1. Baroreflex Mechanism 


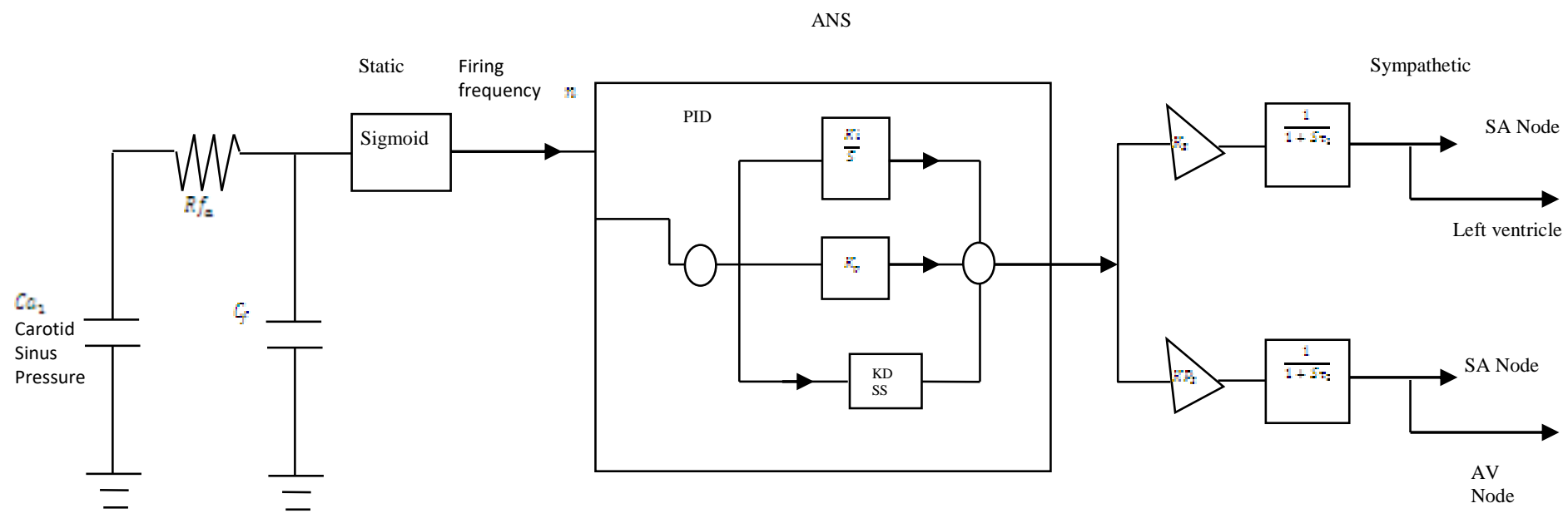

Figure 3.2. Baroreceptor Block Diagram

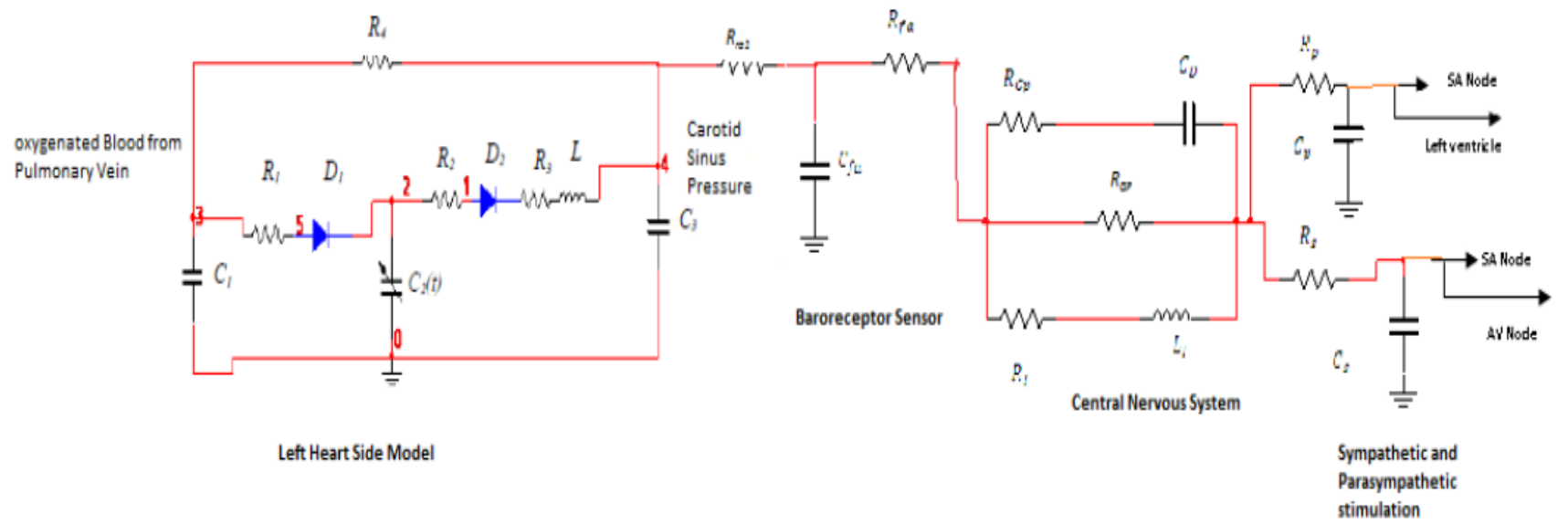

Figure 3.3. Electrical Equivalent Circuit of Baroreceptor Mechanism

A $4^{\text {th }}$ order lumped parameter Wind Kessel model as shown in Figure 3.3 is selected from Shao Hui Chen work [5], which can reflect the left ventricular hemodynamic of the left heart. The parameters and numerical values of the left heart model is given below in Table 1.

\section{Table 1. Parameters of the Left Heart Model}

\begin{tabular}{|l|l|l|}
\hline Parameter & Physiological meaning & Value \\
\hline$C_{1}$ & Left atrial compliance & $4.4 \mathrm{ml} / \mathrm{mm} \mathrm{Hg}$ \\
\hline$C_{2}(t)$ & Time varying left ventricular compliance & Time varying \\
\hline$C_{2}$ & Systemic compliance & $1.333 \mathrm{ml} / \mathrm{mm} \mathrm{Hg}$ \\
\hline$L$ & Inertance of blood in aorta & $0.0005 \mathrm{~mm} \mathrm{Hg.s} / \mathrm{ml}$ \\
\hline$R_{1}$ & Mitral valve resistance & $0.0050 \mathrm{~mm} \mathrm{Hg.s} / \mathrm{ml}$ \\
\hline$R_{2}$ & Aortic valve resistance & $0.0010 \mathrm{~mm} \mathrm{Hg.s} / \mathrm{ml}$ \\
\hline$R_{3}$ & Characteristic resistance & $0.0398 \mathrm{~mm} \mathrm{Hg.s} / \mathrm{ml}$ \\
\hline$R_{4}$ & Systemic vascular resistance & $1.00 \mathrm{~mm} \mathrm{Hg.s} / \mathrm{ml}$ \\
\hline$D_{1}$ & Mitral valve & Ideal switch \\
\hline$D_{2}$ & Aortic valve & Ideal switch \\
\hline
\end{tabular}


The parameter specification and numerical values of the baroreceptor and CNS model is given in Table 3-2.

Table 2. Parameter Values of Baroreflex Model

\begin{tabular}{|c|c|c|c|c|}
\hline Variable & Definition & Value & Units & Reference \\
\hline$C_{f a}$ & $\begin{array}{l}\text { Baroreceptor membrane } \\
\text { capacitance }\end{array}$ & 0.6766 & Farad & [10] \\
\hline$R_{f a}$ & Baroreceptor membrane Resistance & 3.068 & Ohms & [10] \\
\hline$C_{D}$ & $\begin{array}{l}\text { Autonomic nervous system } \\
\text { (Medulla oblongata) derivative } \\
\text { controller capacitance }\end{array}$ & 0.5 & Farad & [19] \\
\hline$R_{G p}$ & $\begin{array}{l}\text { Proportional gain of the Autonomic } \\
\text { nervous system (Medulla } \\
\text { oblongata) }\end{array}$ & 2 & Ohms & [19] \\
\hline$R_{I}$ & $\begin{array}{l}\text { Proportional gain of the Integral } \\
\text { resistance }\end{array}$ & 2 & Ohms & [28] \\
\hline$L_{I}$ & $\begin{array}{l}\text { Proportional gain of the } \\
\text { Integral Inertance }\end{array}$ & 16 & Henry & [32] \\
\hline$R_{\mathrm{p}}$ & $\begin{array}{l}\text { Parasympathetic membrane } \\
\text { resistance }\end{array}$ & 1 & Ohms & [19] \\
\hline$C_{\mathrm{p}}$ & $\begin{array}{l}\text { Parasympathetic membrane } \\
\text { capacitance }\end{array}$ & 0.25 & Farad & [19] \\
\hline$R_{g}$ & Sympathetic membrane resistance & 0.15 & Ohms & [22] \\
\hline$C_{z}$ & Sympathetic membrane capacitance & 1 & Farad & [18] \\
\hline
\end{tabular}

The proposed baroreflex model is simulated in MATLAB and the following results are obtained.

\section{Results, Validation and Discussion}

\subsection{First Subsection; Left Heart Model}

This section corresponds to the left side of heart, proposed and simulated to obtain the time varying Left Ventricular Pressure (LVP), which is taken equal to carotid sinus pressure. The graph obtained is given below in figure. The results can be verified with JCN 8 guide lines for hypertension with systolic pressure $110 \mathrm{mmHg}$ and systolic time 0.34 seconds. 


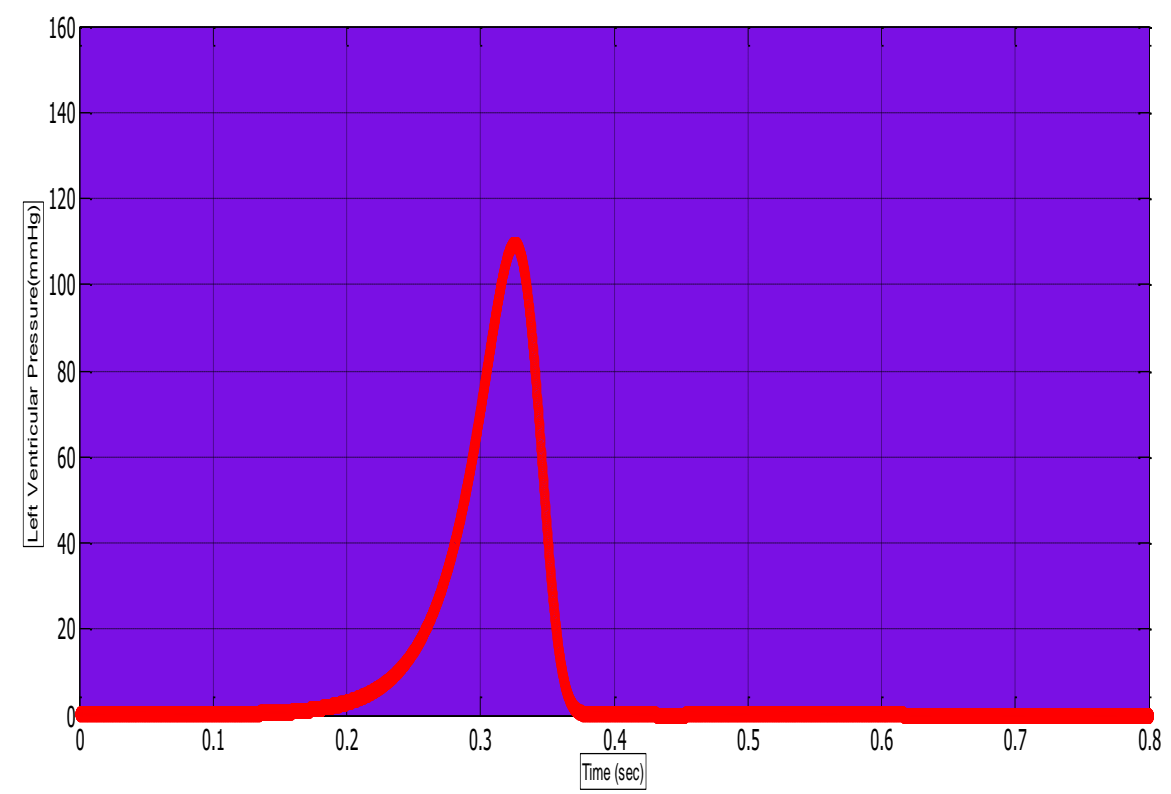

Figure 3.4. Carotid Sinus Pressure

\subsection{Second Subsection; Baroreceptor Model}

The second subsection corresponds to the baroreceptor nerve ending model. The baroreceptors are sensitive to the stretch of the carotid arterial wall which changes with changes in arterial pressure. Due to the complex composition of the arterial wall, the baroreceptor response is non linear. The output from our baroreceptor proposed model is same as given in Gyton [27] given in Figure 3.5.

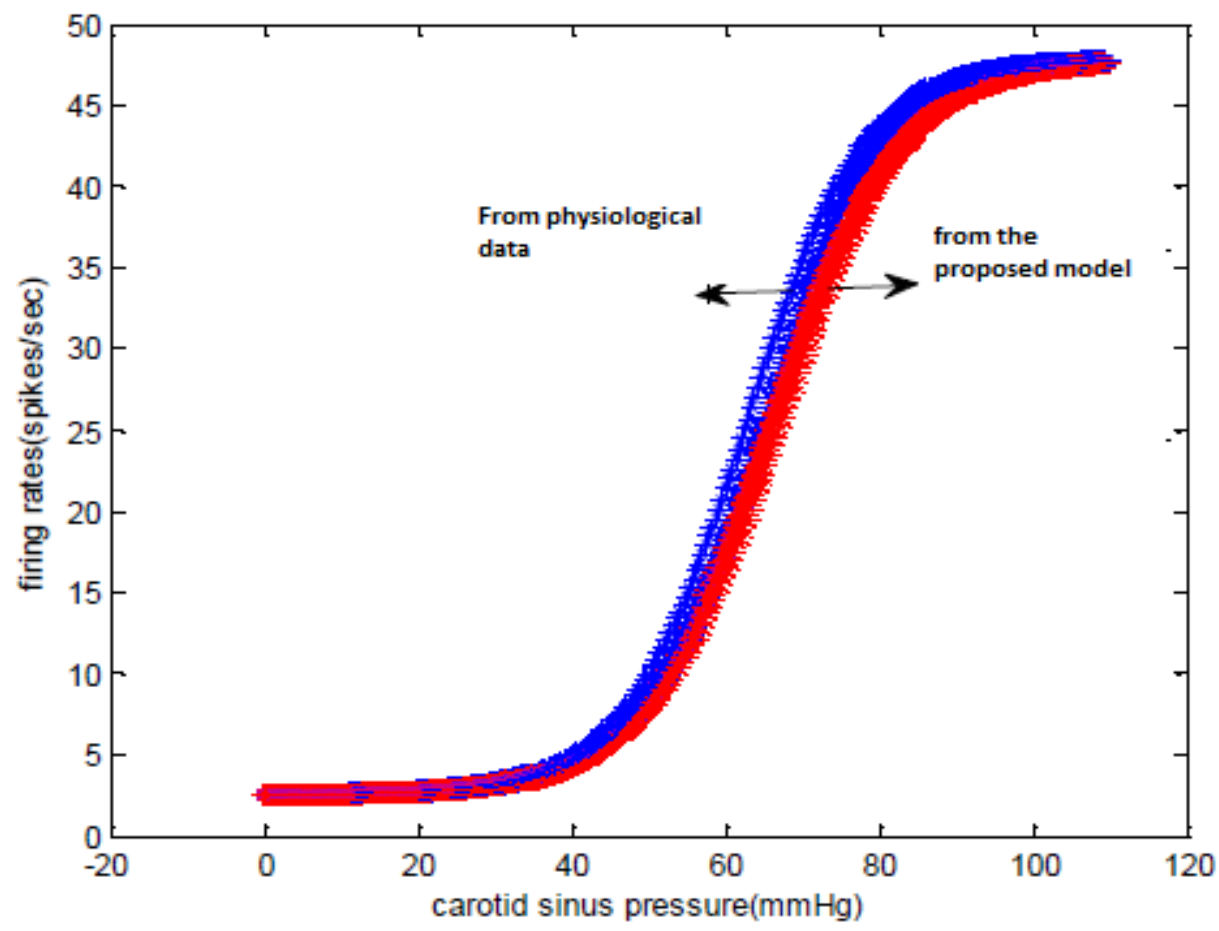

Figure 3.5. Baroreceptor Activation 


\subsection{Third Subsection; Central Nervous System}

This sub model describes effects of the afferent baroreceptor nerve activity on efferent parasympathetic and sympathetic responses. Upon receiving the signals from baroreceptors, the Nucleus of Tractus Solitarius NTS; present in the medulla of CNS will decide either to increase or decrease the heart rate. The glossopharyngeal nerve is connected to Nucleus of Tractus Solitarius NTS present in medulla of CNS. So when the NTS sensed higher firing rates of the glossopharyngeal nerve it does three main function:

1. It stimulates the Cardiac Inhibitory Centre (CIC). This CIC contain vagus nerves; right vagus and left vagus; right vagus carry information to SA node while the left to the AV node with specialized neuron. Parasympathetic stimulation originates from the cardio inhibitory region with impulses travelling via vagus nerves. Parasympathetic stimulation releases neurotransmitter acetylcholine at the neuromuscular junction. This hormone slows down HR by opening potassium ion channel hence slowing down the rate of spontaneous depolarization [30].

2. It also inhibits Cardio Accelerator Centre (CAC) which restricts the sympathetic stimulation by decreasing the release of neurotransmitter norepinephrine, which cause slowing down the rate of depolarization and contraction and hence the rate is decreased [26].

3. It also inhibits the Vasomotor. The specialized sympathetic neurons follow the signals from vasomotor center towards the arterial side, venous side and adrenal medulla, releasing epinephrine and norepinephrine, responsible for increment of rate of depolarization and hence heart rate. Therefore, if the vasomotor is inhibited sympathetic activity is inhibited resulting in the decrease of HR [29].

The signals generated from the proposed CNS model depict the following result, shown in figure below. It should to worth noting that the following result is to increase the heart rate hence parasympathetic is suppressed and sympathetic stimulation is activated.

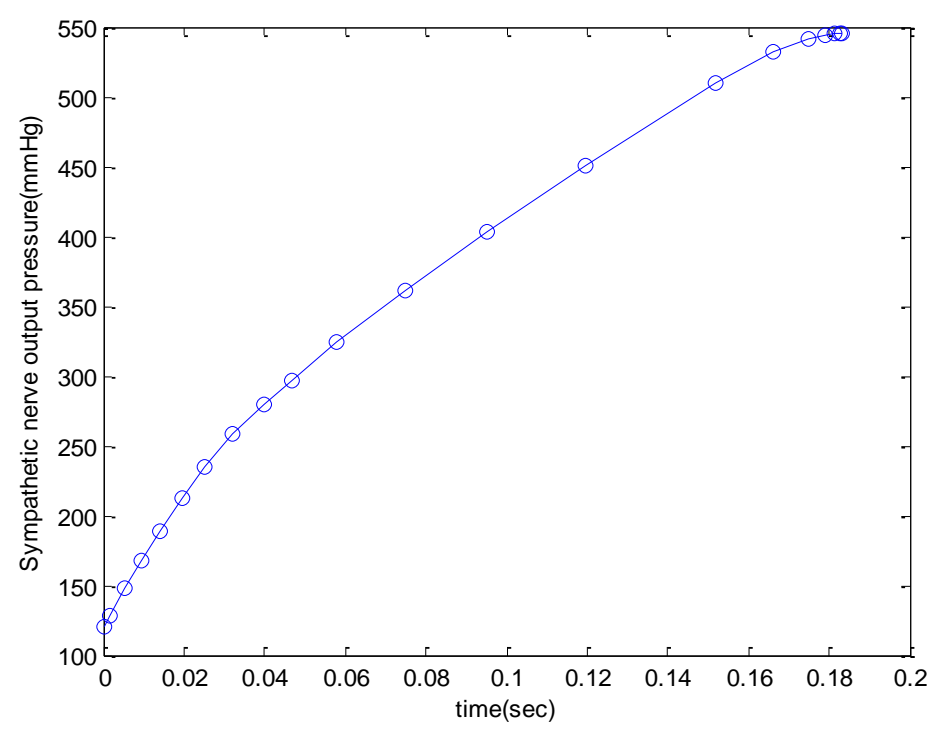

Figure 3.6. Sympathetic Output Pressure vs. Time 


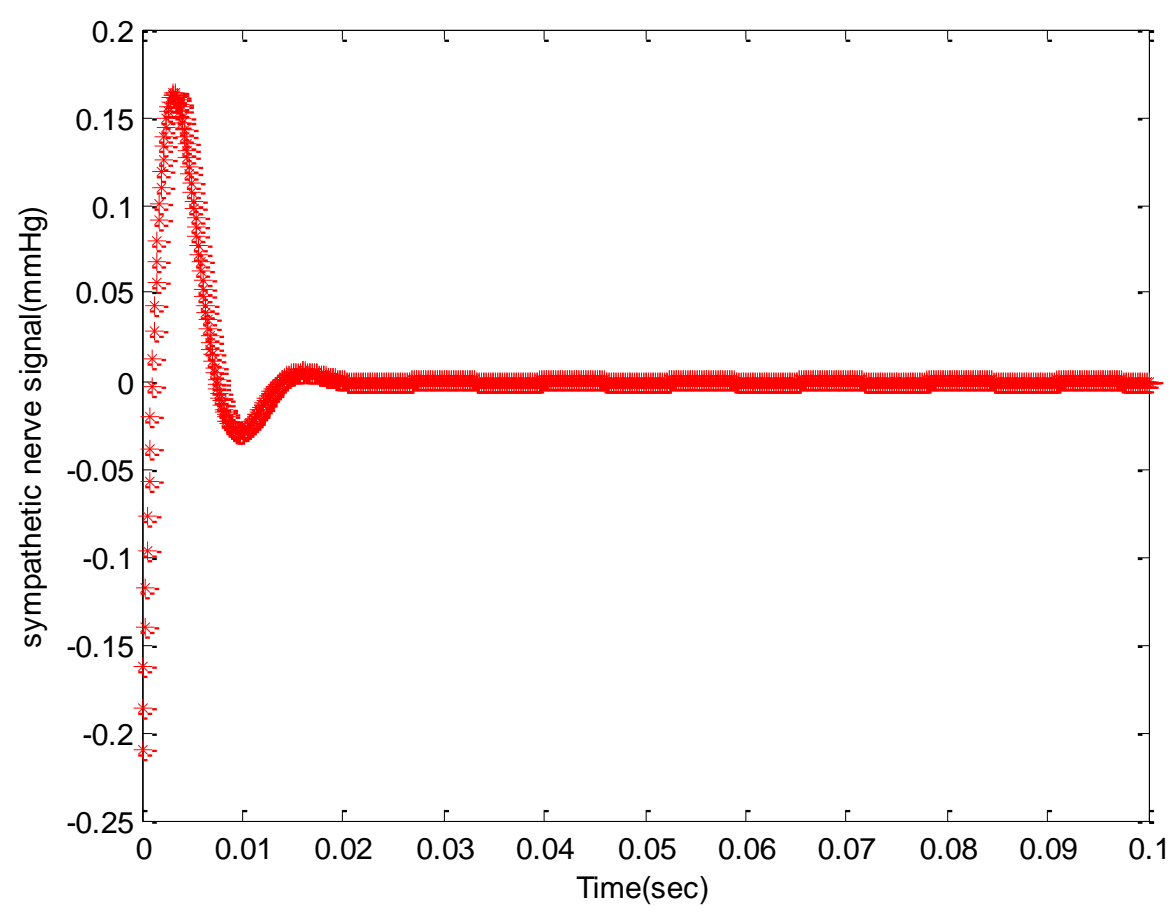

Figure 3.7. Sympathetic Stimulation

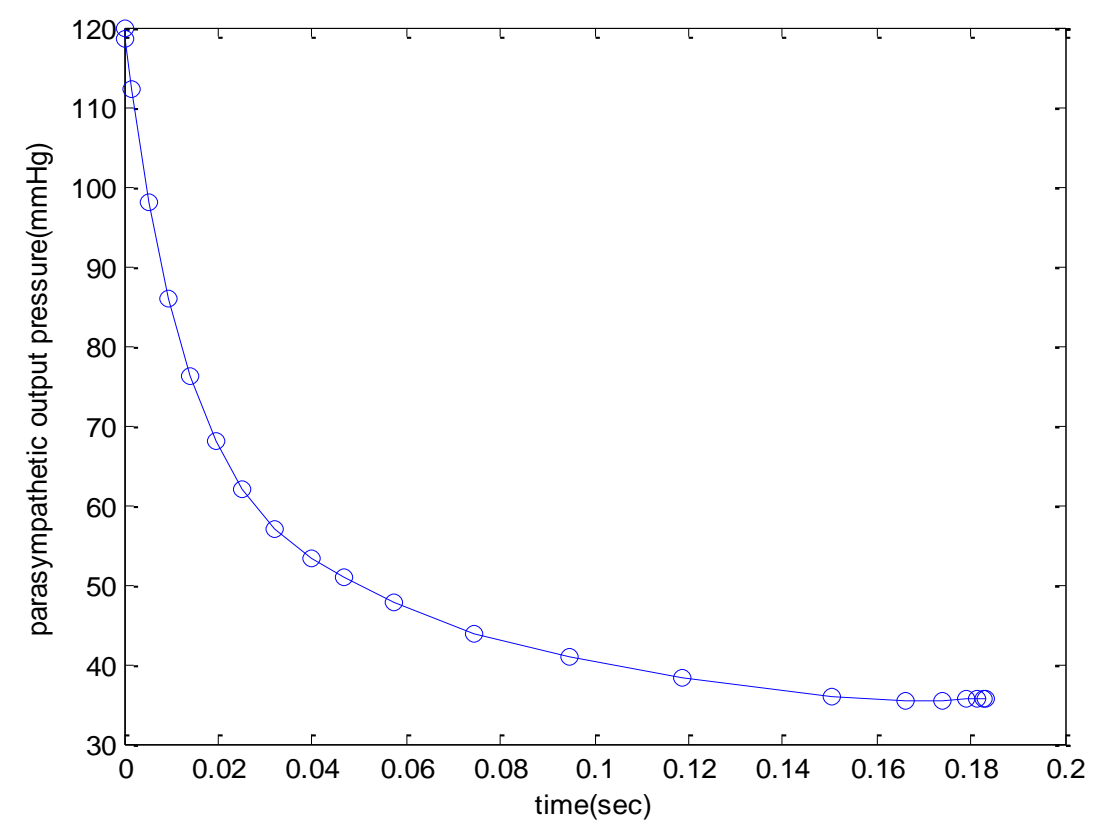

Figure 3.8. Parasympathetic Pressure vs. Time

\section{Conclusion}

The main contribution of the authors to the already existing work is the proposal of a complete baroreflex mechanism electrical equivalent circuit. Representation and simulation of the baroreflex system is a new innovation in this field. The proposed baroreflex model is represented by 3 submodels. One defining the left heart model 
generating the carotid sinus pressure, second portrays the afferent path consisting of first order derivative and a sigmoid function, the second sub model is of PID controller with derivative and integral gains. This sub model portrays the CNS sympathetic and parasympathetic pathways. The sympathetic and parasympathetic pathways then further proceed constituting the efferent part consisting of SA node, AV node, left and right ventricle. All these three subsections are clearly explained through electrical basic components such as capacitor, inductors, resistors, diodes, integral and derivative gains. The results obtained showed a good match from the existing physiological literature. No doubt our model lacks in many aspects such as it does not considers the detail effect of alpha and beta receptors or the effect of $\mathrm{CO}_{2}-\mathrm{O}_{2}$ is also not discussed. Therefore, there is a space for further advancement in future the model.

\section{Appendix}

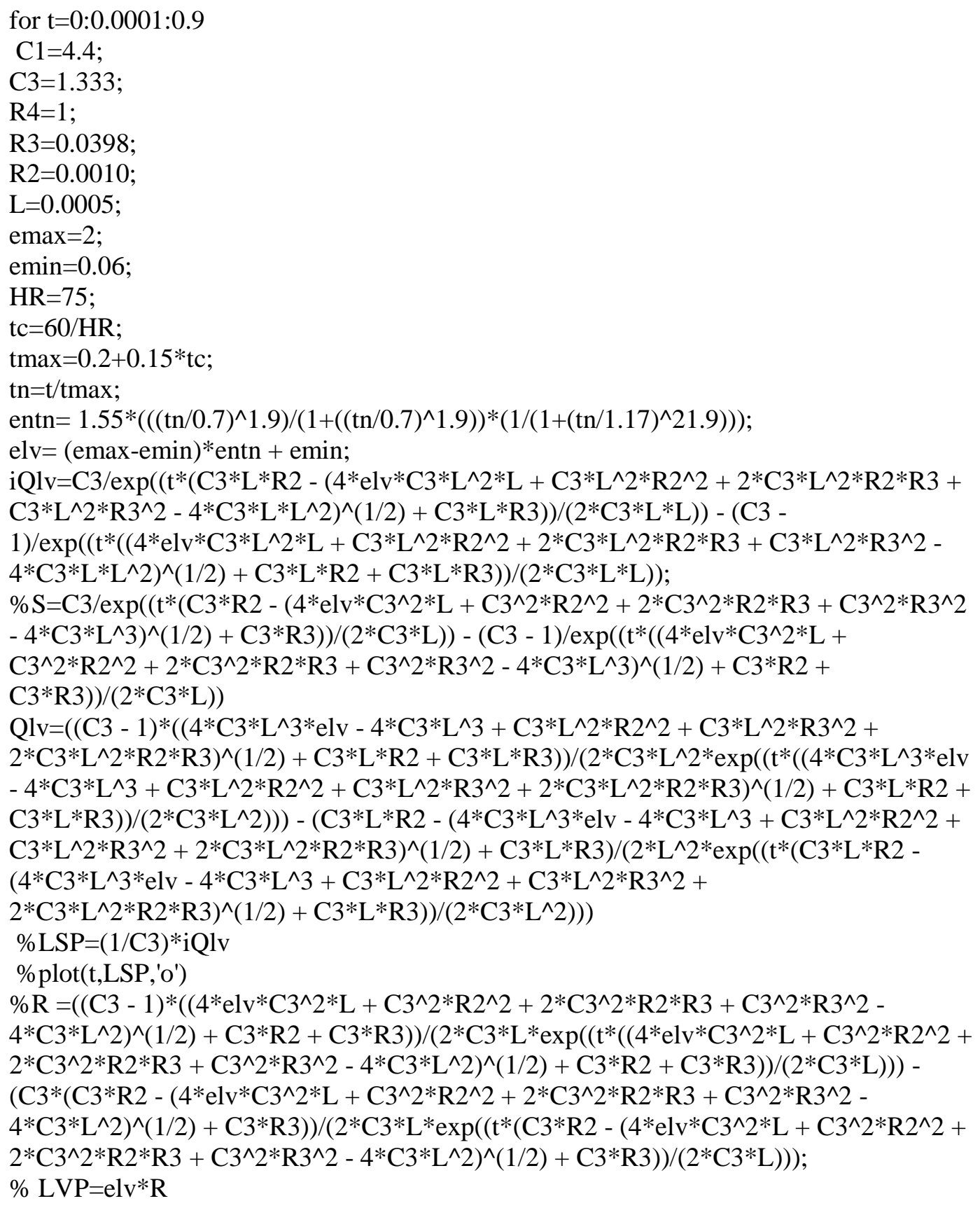




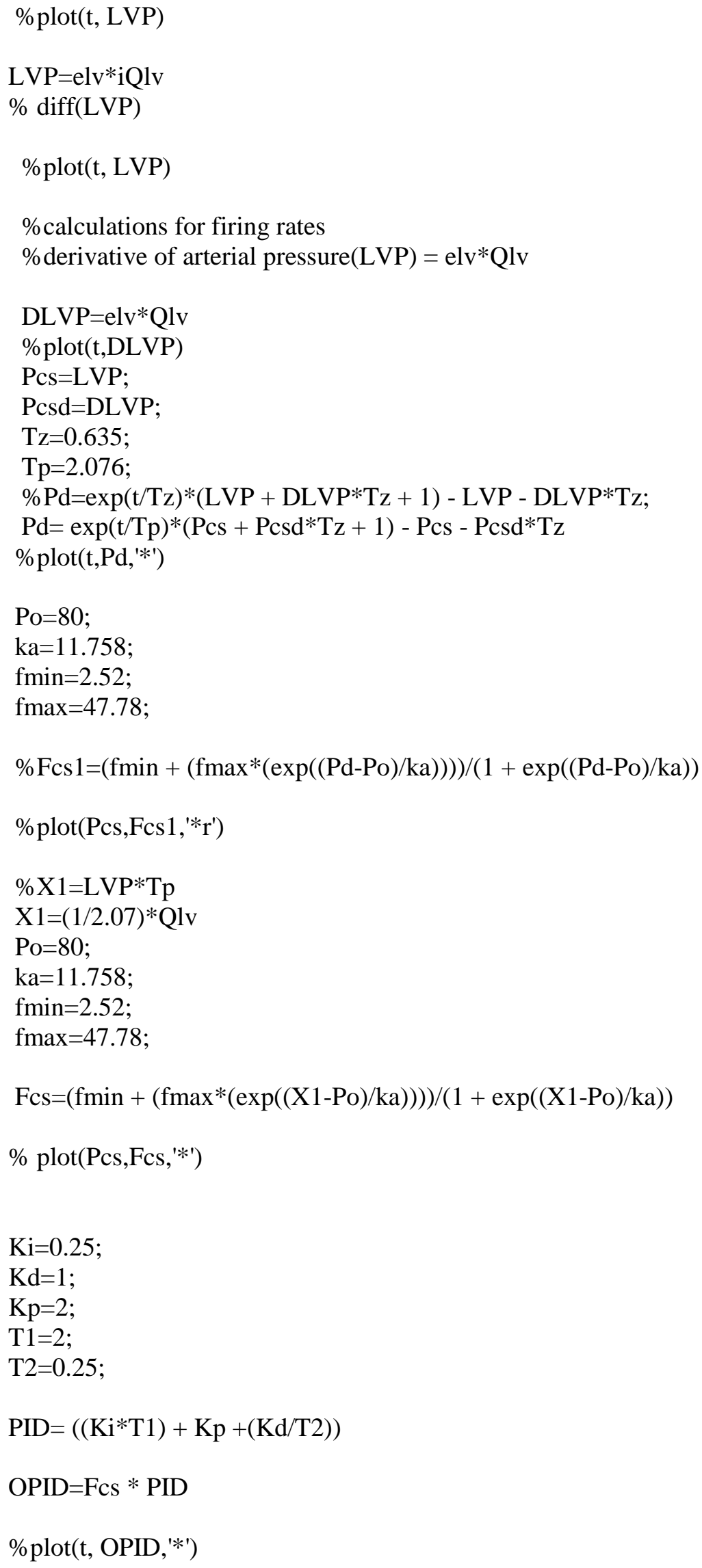




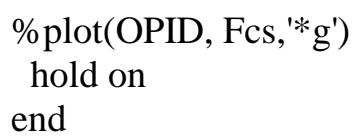

\section{Acknowledgments}

We are grateful to National Institute of Cardio Vascular Diseases NICVD for providing us all required practical information.

\section{References}

[1] M. S. Olufsen, H. T. Tran and J. T. Ottesen, "Modeling baroreflex regulation of heart rate during orthostatic stress", Am J Physiol Regulatory Integrative Comp Physiol, vol. 291, (2006), pp. 1355-1368.

[2] M. Kana, "Mathematical modes of cardiovascular controlled by the autonomic nervous system", Doctoral thesis, Czech technical university, Prague, (2010).

[3] O. Vovkodav and R. Pasichnyk, "The method of identification of a mathematical model for the cardiovascular system response dynamic to exercise stress", Journal of applied science, vol. 22, no. 2, (2014), pp. 91-99.

[4] H. V. D. Vooren and M. G. J. Gademan, "Baroreflex sensitivity, blood pressure buffering and resources, Cenlar simulation of healthy subjects and heart failure patients", Journal Appl Physiol, [Doi: 10.1152/Appl Physiol. 00158], vol. 102, (2006), pp. 1348-1356.

[5] M. Danielsen and J. T. Ottesen, "7. A Baroreceptor Model", Applied Mathematical Models in Human Physiology, [Doi: http://dx.doi.org/10.1137/1.9780898718287.ch7], (2004).

[6] M. Ursino, "Modeling and System Identification", IEEE transactions on Biomedical engineering, vol. 46, no. 4, (1999), pp. 382-392.

[7] M. J. Thubrikar, "Vascular Mechanics and Pathology", Boston, MA: Springer Science +Business Media, LLC, (2006).

[8] A. Mahdi, J. Sturdy, J. T. Ottesen and M. S. Olufsen, "Modeling the Afferent Dynamics of the Baroreflex Control System", Mathematical and computer modeling, [Doi: 10.1371/journal.pcbi.1003384], vol. 9, no. 12, (2013).

[9] R. Srinivasan and H. B. Nudelman, "Modeling the Carotid Sinus Baroreceptor", Biophysics journal, Elsevier, vol. 12, (1972).

[10] Silvan, E. Magosso, S. Bastianini, P. Lenzi and M. Ursino, "Mathematical modeling of cardiovascular coupling: Central autonomic commands and baroreflex control", (2011), [Doi: http://dx.doi.org/10.1016/j.autneu.2011.04.006].

[11] J. T. Ottesen, "Modelling the dynamical baroreflex-feedback control", Mathematical and Computer Modeling, Elsevier, Proceedings of the Conference on Dynamical Systems in Biology and Medicine, [Doi:10.1016/S0895-7177(00)00035-2], vol. 31, iss. 4-5, (2000), pp. 167-173.

[12] W. H. Levison, G. O. Barnett and W. D. Jackson, "Nonlinear Analysis of the Baroreceptor Reflex System", Circulation Research, [Doi:10.1161/01.RES.18.6.673], vol. 18, (1996), pp. 673-682.

[13] L. Bernardi, C. Passino, C. Porta, E. Anesi, G. Palladini and G. Merlini, "Widespread cardiovascular autonomic dysfunction in primary amyloidosis: does spontaneous hyperventilation have a compensatory role against postural hypotension?", Am J Physiol. 274:H1023-31, (1998).

[14] B. Spronck, E. G. Martens, E. D. Gommer and F. N. van de Vosse, "A lumped parameter model of cerebral blood flow control combining cerebral autoregulation and neurovascular coupling", Am Journal Physiol Heart Circ Physiol., H1143-53. doi: 10.1152/ajpheart2012, vol. 303, no. 9.

[15] E. Magosso, S. Cavalcanti and M. Ursino, "Theoretical analysis of rest and exercise hemodynamics in patients with total cavopulmonary connection", Am Journal Physiol Heart Circ Physiol., vol. 282, no. 3, H1018-34, (2002).

[16] G. Mader, M. Olufsen and A. Mahdi, "Modeling Cerebral Blood Flow Velocity During Orthostatic Stress", Annals of biomedical engineering, (2015), pp. 1748-1758.

[17] A. Mahdi, J. T. Ottesen and M. Olufsen, "Qualitative features of a new baroreceptor model", Proceeding of the 1st international workshop on innovative simulation for healthcare, (2012), pp. 75-80.

[18] C. Scholzel, A. Goesmann, G. Ernst and A. Dominik, "Modeling Biology in Modelica: The Human Baroreflex", Proceedings of the 11th International Modelica Conference, [Doi: 10.3384/ecp15118367], (2015).

[19] M. D. I. Rienzo, G. Parati, A. Radaelli and P. Castiglioni, "Baroreflex contribution to blood pressure and Heart rate oscillations: time scales, time-variant characteristics and nonlinearities", Phil. Trans. R. Soc. A, vol. 367, (2009), pp. 1301-1318.

[20] J. Batzel, G. Baselli, R. Mukkamala and K. H. Chon, "Modeling and disentangling physiological mechanisms: linear and nonlinear identification techniques for analysis of cardiovascular regulation", Phil. Trans. R. Soc., [Doi:10.1098/rsta.2008.0266], vol. 367, (2009), pp. 1377-1391. 
[21] S. Lakshmi and M. Senbagavalli, "Mathematical model for finding the steadystate and transient behavior of the secretion of vasopressin in hypertension cases", IJMS, vol. 11, no. 1-4, (2012), pp. 393399.

[22] S. Gisler, "Modelling and control of the human cardiovascular system", Phd Thesis Sensory Motor Systems Lab (SMS), Swiss Federal Institute of Technology Zurich (ETH), (2011).

[23] V. McLoone, "Modelling of Long and Short Term Blood Pressure Control Systems", Phd thesis, Nui Maynooth, (2014).

[24] E. Gao, W. L. Young, J. Pile-Spellman, E. Ornstein and Q. Ma, "Mathematical considerations for modeling cerebral blood flow autoregulation to systemic arterial pressure", IEEE transactions on biomedical engineering, vol. 46, no. 4, (1999).

[25] E. Lim, "A Cardiovascular Mathematical Model of Graded Head-Up Tilt", PLOS 1, DOI: 10.1371/journal.pone.0077357, (2013).

[26] Cardiovascular physiology Concepts by Dr. Richard Klabunde, second edition,ISBN: 9781451113846. Published by Lippincott Williams \& Wilkins, (2011).

[27] Textbook of Medical Physiology by Guyton and Hall, 13 ${ }^{\text {th }}$ edition, ISBN:978-1-4557-7005-2, Elsevier, (2016).

[28] Neuroscience by Sunderland, $2^{\text {nd }}$ edition,Sinauer Associates, ISBN: 0-87893-742-0, (2001).

[29] Autonomic Neural Control of Cardiac Function by Matthew N. Levy, Paul J. Martin, ISBN 978-14612-8222-8, 10.1007/978-1-4613-0873-7_17, Springer, (1989).

[30] Principles of Cardiovascular Neural Regulation in Health and Disease by John Longhurst, Norwell, Mass: Kluwer Academic Publishers; ISBN 0-7923-7775-3, (2000). 
International Journal of Bio-Science and Bio-Technology

Vol.9, No.1 (2017) 